\title{
DEVELOPTMENT OF IN SITU SOFT X-RAY ABSORPTION SPECTROSCOPIC TECHNIQUE UNDER HIGH TEMPERATURE AND CONTROLLED ATMOSPHERE
}

Ryo Oike, Koji Amezawa*, Takashi Nakamura

IMRAM, Tohoku University, 2-1-1 Katahira, Aoba, Sendai 980-8577, Japan

Yusuke Tamenori

JASRI, 1-1-1, Kouto, Sayo-gun, Hyogo 679-5198, Japan

Keiji Yashiro, Tatsuya Kawada

Grad. Sch. Environmental Studies, Tohoku University, 6-6-01, Aramaki, Aoba, Sendai 980-8579, Japan

*Corresponding author: amezawa@tagen.tohoku.ac.jp

\section{Abstract}

In this study, in situ soft X-ray absorption spectroscopy (soft XAS) technique, which enables us to analyzeelectronic structures of oxides at elevated temperatures while controlling atmospheric conditions, was developed. The technique was applied to investigate the electronic structures of $\mathrm{La}_{0.6} \mathrm{Sr}_{0.4} \mathrm{CoO}_{3-\delta}$. X-ray absorption spectra at the $\mathrm{Co} L$-edges and the $\mathrm{O}$ 
$K$-edge were measuredin the temperature range from room temperature to $873 \mathrm{~K}$ and the $p\left(\mathrm{O}_{2}\right)$ range from $10^{-4}$ to $10^{-2}$ bar.The developed in situsoft XAS technique is found to be effective for evaluating electronic structures of oxidesunder controlled temperature and $p\left(\mathrm{O}_{2}\right)$.

Keywords: Soft X-ray, X-ray absorption spectroscopy, in situ, high temperature

\section{Introduction}

Ion/electron conducting oxides are widely utilized in electrochemical devices such as solid oxide fuel cells (SOFCs), lithium-ion secondary batteries and so on. In order to improve the device performance, it is essential to understandelectronic structures of the components, which are often related deeply to their electrical and electrochemical properties. For the analysis of electronic structures of oxides, X-ray absorption spectroscopy (XAS) is frequentlyapplied.XAS is a spectroscopic analytical technique detecting the excitation of core electrons by the X-ray irradiation, and is available to examine unoccupied orbitals of materials. In the case of $3 \mathrm{~d}$ transition metal oxides, hybrid orbitals betweenthe transition metal $3 \mathrm{~d}$ and oxygen $2 \mathrm{p}$ orbitalsare known to be responsible for their electronic properties. When XAS is applied to investigate electronic states of those orbitals, it is required to measure X-ray absorption at the $3 \mathrm{~d}$ transition metal $L_{\mathrm{II}}$ and $L_{\mathrm{III}}$-edges and the $\mathrm{O} K$-edge, which give us information on electronic structures of the transition metal $3 \mathrm{~d}$ and oxygen $2 \mathrm{p}$ orbitals, respectrively. 
Energies of above absorption edges range between 500 and $900 \mathrm{eV}$, i.e. in the soft X-ray region. Since soft X-ray is easily absorbed even by gases such as $\mathrm{N}_{2}, \mathrm{O}_{2}, \mathrm{H}_{2} \mathrm{O}$ and so on, it is difficult for X-ray to penetrate the long distance in atmospheres containing above gases. Thus, in general, soft XAS measurements are carried out in high vacuum conditions. However, measurements in high vacuum conditions are not desirable for the analysis of oxides, because oxides can change their oxygen nonstoichiometry and electronic structures depending on atmospheric $p\left(\mathrm{O}_{2}\right)[1]$.

In order to avoid such problems, in this work, we developed in situ soft XAS technique which can be used under desired temperature and atmospheric conditions. $\mathrm{La}_{0.6} \mathrm{Sr}_{0.4} \mathrm{CoO}_{3-\delta}$ having the perovskite-type structure was chosen as a model oxide. $\mathrm{La}_{1-x} \mathrm{Sr}_{x} \mathrm{CoO}_{3-\delta}$ is a candidate of $\mathrm{SOFC}$ cathode materials because of their high electronic/ionic conductivity and high catalytic activity for the reductionof oxygen gas[2, 3].XAS spectra at the Co $L_{\mathrm{II}}$ and $L_{\mathrm{III}}$-edges and the $\mathrm{O} K$-edge were measured under $p\left(\mathrm{O}_{2}\right)=$ $10^{-4}-10^{-2}$ bar at RT $-873 \mathrm{~K}$, and validity of the developed technique was confirmed.

\section{Experimental}

\subsection{Sample preparation}

For the preparation ofa dense compact of $\mathrm{La}_{0.6} \mathrm{Sr}_{0.4} \mathrm{CoO}_{3-\delta}$, the commercially available powder (AGC SEIMI CHEMICAL Co., Ltd.) was uniaxially pressed in a cylindrical shape, and was sintered at $1473 \mathrm{~K}$ for 4 hours. The relative density of the obtained compact 
washigher than $92 \%$. The compact was cut into a plate shape of $5 \times 3 \times 0.8 \mathrm{~mm}$ and polished with the diamond paste of $3 \mu \mathrm{m}$. Before XAS measurements, the $\mathrm{La}_{0.6} \mathrm{Sr}_{0.4} \mathrm{CoO}_{3-\delta}$ compact was annealed at $973 \mathrm{~K}$ for 2 hours and slowly cooled down to $473 \mathrm{~K}$ for 4 days under $1 \% \mathrm{O}_{2}$-Ar gas.

\subsection{Soft X-ray absorption spectroscopy}

Soft XAS measurements were performed at the beam line of BL27SU at SPring-8, JASRI, Japan. The system for soft XAS under controlled atmosphere at elevated temperature was developed based on the differential pumping system for soft XAS under normal atmospheric conditions by Tamenori [4]. In order to control partial gas pressures while ensuring the sufficient incident X-ray, the sample chamber was separated from the optical upstream part with an $\mathrm{Si}_{3} \mathrm{~N}_{4}$ thin film window of $500 \mathrm{~nm}$. To achieve in-situsoft XAS measurements at elevated temperature, a special sample holder was designed and fabricated.In this holder, the Pt-Ir electric furnace was used to heat the sample and the sample was placed inside the furnace toreducethe thermal noises from the furnace as much as possible. The specimen was tilted at a 45 degrees angle to the incidence X-ray and the fluorescence X-ray detector, so that the incident X-ray could be delivered to wider area on the sample surface and the exited fluorescence X-ray was detected efficiently. Inside the sample chamber, the mixture of He and $\mathrm{O}_{2}$ gases was flowed to control $p\left(\mathrm{O}_{2}\right)$ around the sample. In order to minimize the X-ray absorption by gases, the light passes in the sample chamber were designed to be as short as 
possible.

In this work, soft XAS measurements of $\mathrm{La}_{0.6} \mathrm{Sr}_{0.4} \mathrm{CoO}_{3-\delta}$ at $\mathrm{Co} L_{\mathrm{II}}-(\sim 795 \mathrm{eV})$ and $L_{\mathrm{III}}$-edge $(\sim 780 \mathrm{eV})$ and $\mathrm{O} K$-edge $(\sim 530 \mathrm{eV})$ were performed with the fluorescence mode by using a photodiode as a detector. Temperature was controlled from room temperature to 873 K.Oxygen partial pressure was controlled from $10^{-4}$ to $10^{-2}$ bar, and was monitored by a zirconia oxygen sensor at the outlet from the sample chamber.

\section{Results and Discussion}

X-ray absorption spectra of $\mathrm{La}_{0.6} \mathrm{Sr}_{0.4} \mathrm{CoO}_{3-\delta}$ atthe $\mathrm{Co} L$-edges and $\mathrm{O} K$-edge were measured under $p\left(\mathrm{O}_{2}\right)=10^{-4}-10^{-1}$ bar at various temperatures. Fig. 1 shows the absorption spectra of $\mathrm{La}_{0.6} \mathrm{Sr}_{0.4} \mathrm{CoO}_{3-\delta}$ at the $\mathrm{Co} L$-edges under $p\left(\mathrm{O}_{2}\right)=10^{-4}-10^{-1}$ bar at room temperature. The spectra were normalized by the peak height at $780 \mathrm{eV}$. Absorption spectra were clearly observed under $p\left(\mathrm{O}_{2}\right)=10^{-4}-10^{-2}$ bar. On the other hand, spectra could not be observed under $p\left(\mathrm{O}_{2}\right)=10^{-1}$ bar. In the case of X-ray of $780 \mathrm{eV}$, if we assume $5 \mathrm{~cm}$ of the light pass, approximately $55 \%$ of X-ray can penetrate under $1 \% \mathrm{O}_{2}-\mathrm{He}$ mixed gas. However, only $0.3 \%$ of X-ray can penetrate through $5 \mathrm{~cm}$ under $10 \% \mathrm{O}_{2}-\mathrm{He}$ mixed gas. It is thought that such a difference in the penetrating power of soft X-ray against oxygen gasdecides the $p\left(\mathrm{O}_{2}\right)$ limit for in situ soft XAS measurements in our setup.Normalized absorption spectra of $\mathrm{La}_{0.6} \mathrm{Sr}_{0.4} \mathrm{CoO}_{3-\delta}$ at theCo $L$-edgesunder $p\left(\mathrm{O}_{2}\right)=10^{-4}$ bar at various temperatures were shown in Fig. 2.At $873 \mathrm{~K}$, soft XAS measurements were performed two times and X-ray absorption 
spectrum was averagedin order to minimize the influence of the thermal noises.Absorption spectra were clearly observed at temperatures lower than $873 \mathrm{~K}$, while spectra could not be obtained at above $873 \mathrm{~K}$. This is possibly becauseradiation from the furnace became significant with increasing temperature. From the results given in Figs. 1 and 2, it was demonstrated that the in situ XAS technique developed in this work enables us to measure absorption spectra in soft X-ray region while controlling temperature lower than $873 \mathrm{~K}$ and $p\left(\mathrm{O}^{2}\right)$ lower than $10^{-1}$ bar.

In the absorption spectra of $\mathrm{La}_{0.6} \mathrm{Sr}_{0.4} \mathrm{CoO}_{3-\delta}$ at $L_{\mathrm{III}}$-edge shown in Figs. 1 and 2, a shoulder at the lower energy side of the main peak was observed.The absorption of incident X-ray is caused by the transition of the core orbital electron to the unoccupied levels. This means that XAS spectra reflect the spin state and the valence state of the investigated atom. In the case of Co $L_{\mathrm{II}}$ and $L_{\mathrm{III}}$-edges, XAS spectra were associated with the transition of electrons from the Co $2 p$ to the $3 \mathrm{~d}$ state. In $\mathrm{La}_{0.6} \mathrm{Sr}_{0.4} \mathrm{CoO}_{3-\delta}$, the mean valence of cobalt ions is considered as higher than 3. As temperature increases, the mean valence decreases even under the constant oxygen partial pressure, because of the formation of oxygen vacancies[1]. However, in this study, the spectrum shape did not change apparently. The reason for this is possibly because the variation in the mean valence of cobalt ions were negligible, because the amount of formed oxygen vacancies is small under the experimental conditions [5]. Another possible explanation is that the change in the oxygen nonstoichiometry did not considerably affect the 
electronic structures in the Co $3 \mathrm{~d}$ band because the Co $3 \mathrm{~d}$ band is strongly hybridized with the O $2 p$ band [6].

Fig. 3 show the absorption spectra of $\mathrm{La}_{0.6} \mathrm{Sr}_{0.4} \mathrm{CoO}_{3-\delta}$ atthe $\mathrm{O} K$-edge under $p\left(\mathrm{O}_{2}\right)=10^{-4}$ bar at various temperatures.The spectra were normalized to equalize the peak heights at $527 \mathrm{eV}$. At $873 \mathrm{~K}$, soft XAS measurements were performed three times and X-ray absorption spectrum was averaged. The absorption spectra at the $\mathrm{O} K$-edge were associated with the transition of electrons from the $\mathrm{O} 1 \mathrm{~s}$ core level to the unoccupied statesin the $\mathrm{O} 2 \mathrm{p}$ level,hybridized with the orbitals of bonding cations.According to Abbatte et al.,peaks around $527 \mathrm{eV}$ are assigned to the unoccupied orbitals of $\mathrm{O} 2 \mathrm{p}$ orbital hybridized with Co $3 \mathrm{~d}$ orbital[7].

Since the X-ray energy for XAS measurements at the $\mathrm{O} K$-edge is much lower than that at the Co $L$-edge,the absorption of X-ray by atmospheric gases is considered more significant. Nevertheless, it was found that $\mathrm{O} K$-edge absorption spectra could be observed below $773 \mathrm{~K}$ by using in situ XAS system developed in this work. XAS measurement was carried out under atmosphere containing oxygen gas.Thus, absorption spectra at the $\mathrm{K}$-edge would include signals from not only oxide ions in $\mathrm{La}_{0.6} \mathrm{Sr}_{0.4} \mathrm{CoO}_{3-\delta}$ but also oxygen gas and moisture in atmosphere. A small peak at around $530 \mathrm{eV}$, shown by an open triangle, is assigned to oxygen gas, and small peaks at around $533-538 \mathrm{eV}$, shown by closed triangles, are attributed to $\mathrm{H}_{2} \mathrm{O}$ gas [8].However, as shown in Fig. 3, the absorption peaks due to the oxide and the gases were 
separately observed. In particular, the absorption peaks assigned to the hybridized orbitals with the Co $3 \mathrm{~d}$ band, which are strongly related to electronic properties, did not overlap with those assigned to the atmospheric gases. This indicated that the developed technique is applicable for examining electronic structures of oxides, especially functional oxides for high temperature uses.

\section{Conclusions}

Novel in situ soft X-ray absorption spectroscopy technique was developed in this study and applied to investigate electronic structures of $\mathrm{La}_{0.6} \mathrm{Sr}_{0.4} \mathrm{CoO}_{3-\delta}$ under controlled temperature and $p\left(\mathrm{O}_{2}\right)$. It was found out that the developed system enabled us to measure $\mathrm{X}$-ray absorption

spectra in the soft X-ray region, e.g. $500-900 \mathrm{eV}$, under $p\left(\mathrm{O}_{2}\right)$ lower than $10^{-2}$ bar and at temperaturesup to $873 \mathrm{~K}$.The developed technique can be a powerful tool to evaluate electronic structures of functional oxides for high temperature electrochemical devices.

\section{Acknowledgements}

This work was supported by JST-CREST and the Grand-in-Aid for Scientific Research of

MEXT, Japan.

\section{References}

[1] J. Mizusaki, Y. Mima, S. Yamauchi, K. Fueki, Solid State Chem.80 (1989)102-111.

[2]S. B. Adler, Chem. Rev.104(2004) 4791-4844. 
[3]R. M. Ormerod, Chem. Soc. Rev.32 (2003)17-28.

[4] Y. Tamenori, J.Synchrotron Rad.17 (2010)243-249.

[5] M. Kuhn, S. Hashimoto, K. Sato, K. Yashiro, J. Mizusaki, J. Solid State

Chem.197(2013)38-45.

[6] Y. Orikasa, T. Ina, T. Nakao, A. Mineshige, K. Amezawa, M. Oishi, H. Arai, Z. Ogumi, Y. Uchimoto, J. Phys. Chem. C115 (2011)16433-16438.

[7] M.Abbate, J. C. Fuggle, A. Fujimori, L. H. Tjeng, C. T. Chen, R. Potze, G. A. Sawatzky, H. Eisaki, S. Uchida, Phys. Rev. B47 (1993)16124-16130.

[8] C. D. Cappa, J. D. Smith, K. R. Wilson, R. J. Saykally, J. Phys. Condens. Matter.20(2008)205105.

\section{Figure Captions}

Fig. 1:X-ray absorption spectra of $\mathrm{La}_{0.6} \mathrm{Sr}_{0.4} \mathrm{CoO}_{3-\delta}$ at $\mathrm{Co} L_{\mathrm{II}}$ and $L_{\mathrm{III}}$-edgesunder $p\left(\mathrm{O}_{2}\right)=10^{-4}$

$-10^{-1}$ bar at room temperature.

Fig. 2:X-ray absorption spectra of $\mathrm{La}_{0.6} \mathrm{Sr}_{0.4} \mathrm{CoO}_{3-\delta}$ at $\mathrm{Co} L_{\mathrm{II}}$ and $L_{\mathrm{III}}$-edges under $p\left(\mathrm{O}_{2}\right)=10^{-4}$ bar at various temperatures.

Fig. 3:X-ray absorption spectra of $\mathrm{La}_{0.6} \mathrm{Sr}_{0.4} \mathrm{CoO}_{3-\delta}$ at $\mathrm{O} K$-edgeunder $p\left(\mathrm{O}_{2}\right)=10^{-4}$ bar at various temperatures. 


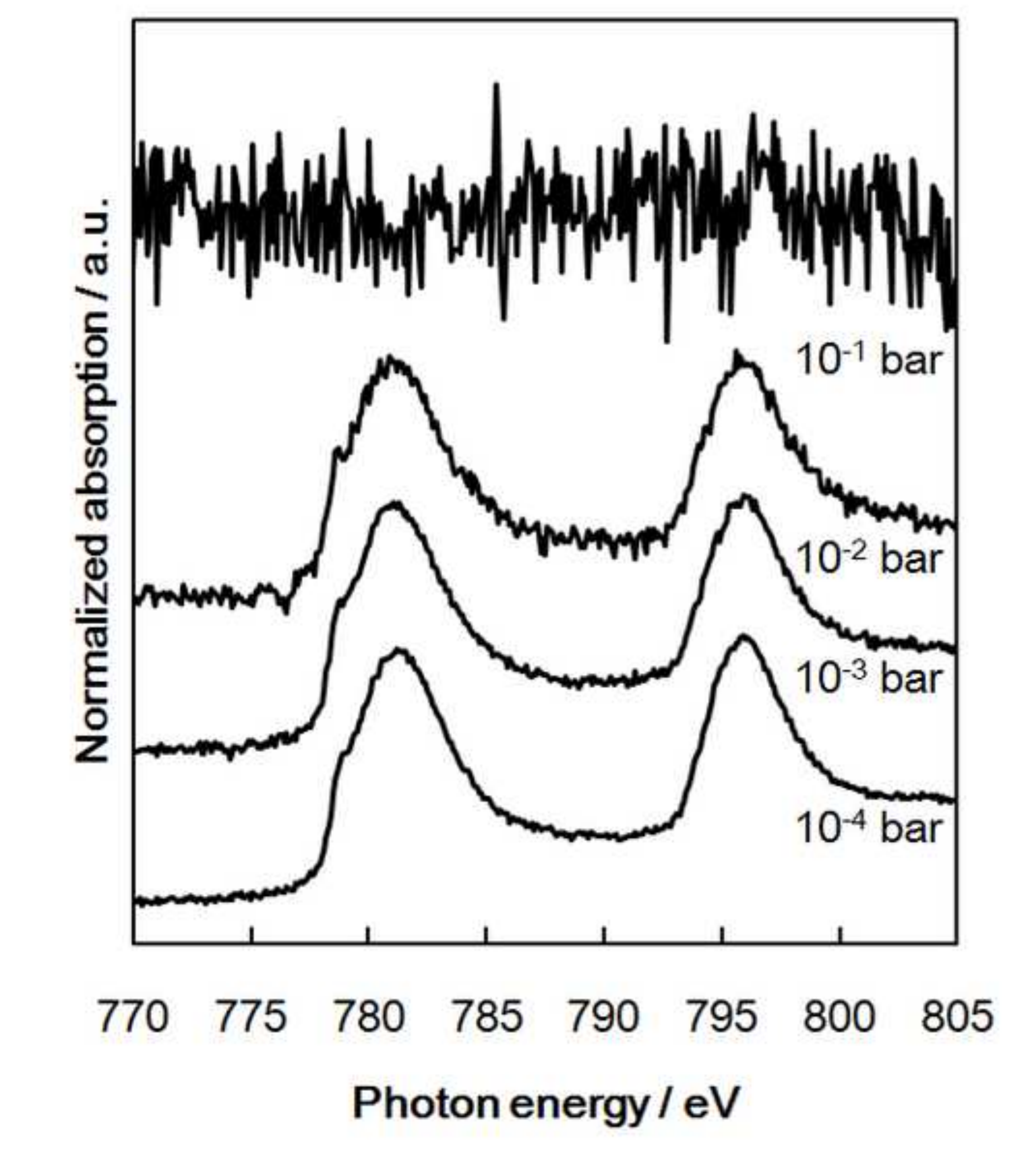

Figure 1

(4)

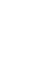




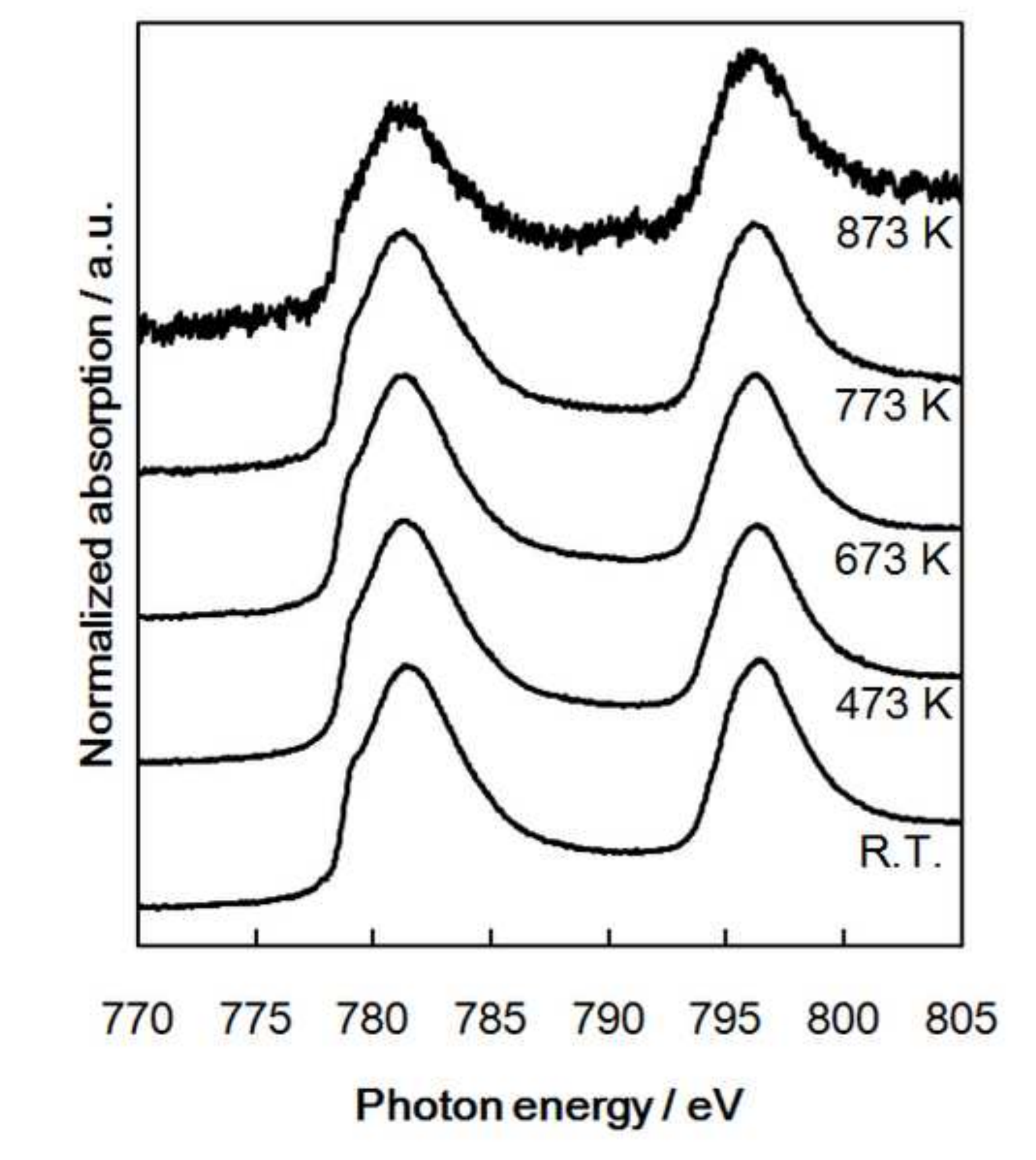

$\begin{array}{llllllll}770 & 775 & 780 & 785 & 790 & 795 & 800 & 805\end{array}$ Photon energy / eV

Figure 2

,

2

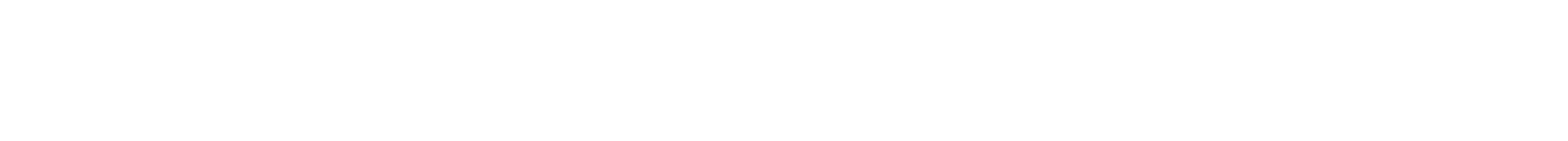


Figure(3)

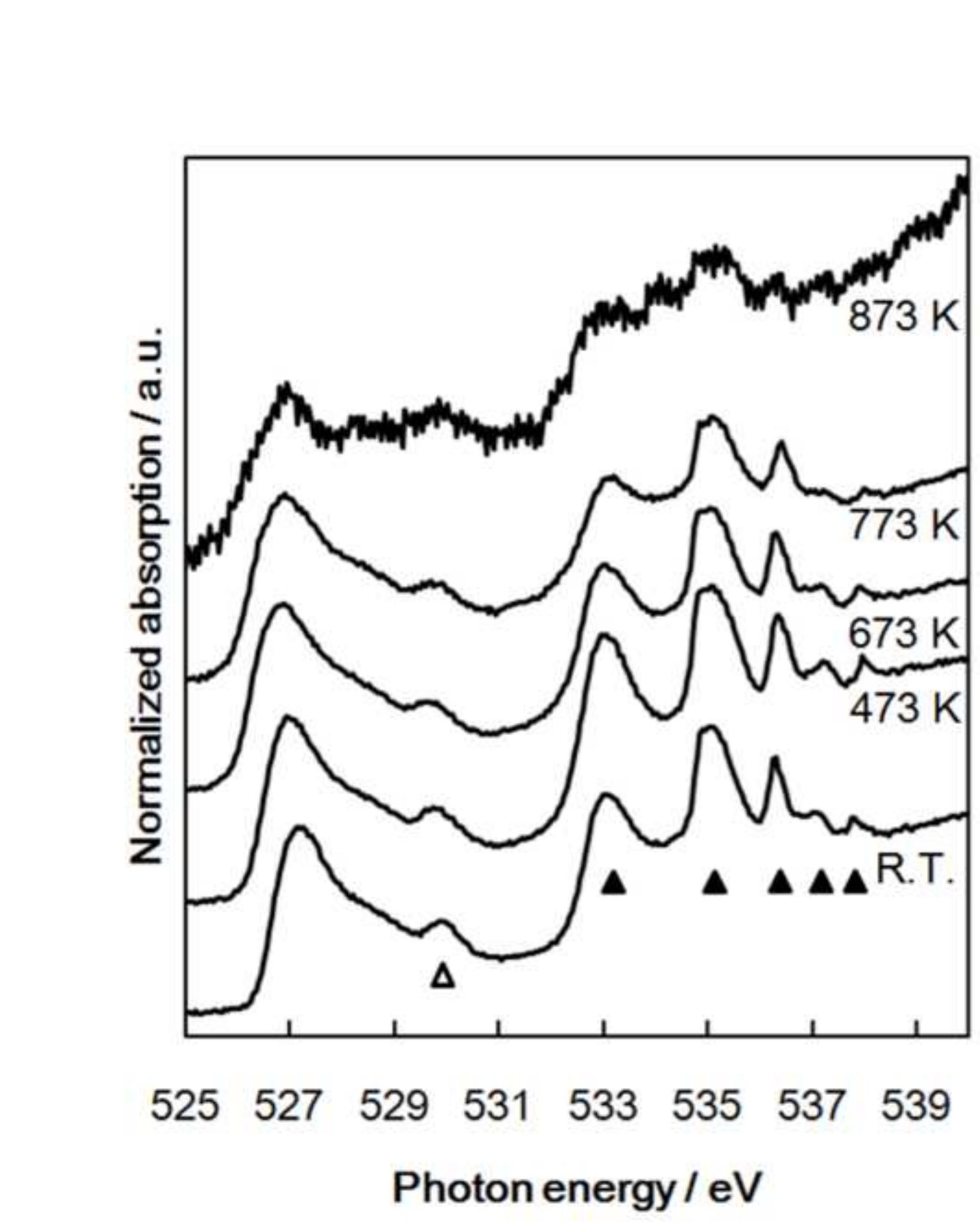

\section{$\begin{array}{llllllll}525 & 527 & 529 & 531 & 533 & 535 & 537 & 539\end{array}$ Photon energy / eV}

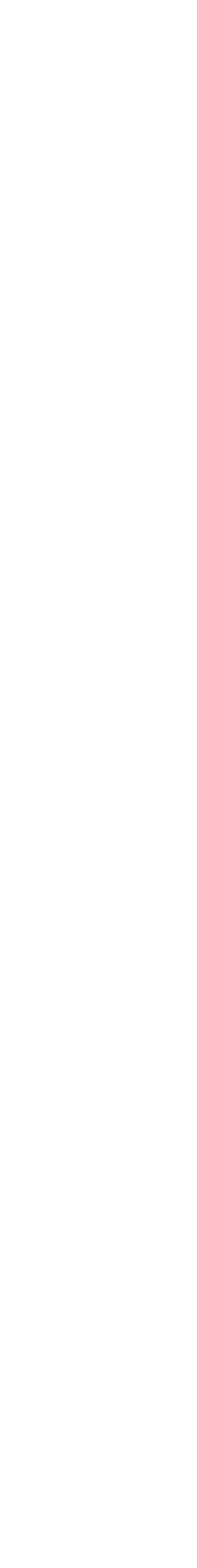

